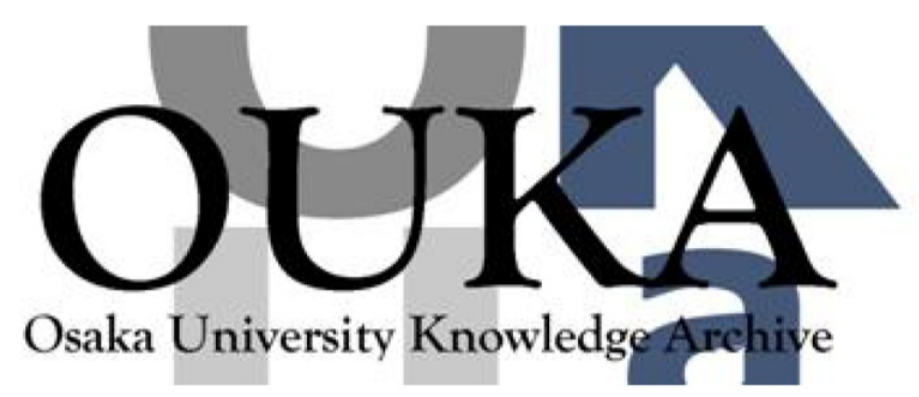

\begin{tabular}{|c|l|}
\hline Title & $\begin{array}{l}\text { Increase of efficiency of magnetostriction SH- } \\
\text { Wave electromagnetic acoustic transducer by } \\
\text { angled bias field : Piezomagnetic theory and } \\
\text { measurement }\end{array}$ \\
\hline Author(s) & 0gi, Hirotsugu; Goda, Eiji; Hirao, Masahiko \\
\hline Citation & $\begin{array}{l}\text { Japanese Journal of Applied Physics. } 42 \text { p. 3020- } \\
\text { p. 3024 }\end{array}$ \\
\hline Issue Date & $2003-05-01$ \\
\hline oaire:version & AM \\
\hline URL & https://hdl. handle. net/11094/84155 \\
\hline rights & \\
\hline Note & \\
\hline
\end{tabular}

Osaka University Knowledge Archive : OUKA

https://ir. Library. osaka-u. ac. jp/

Osaka University 


\section{Increase of Efficiency of Magnetostriction SH-Wave Electromagnetic Acoustic Transducer by Angled Bias Field: Piezomagnetic Theory and Measurement}

Hirotsugu Ogi, Eiji Goda and Masahiko Hirao

Graduate School of Engineering Science, Osaka University, 1-3 Machikaneyama, Toyonaka, Osaka 560-8531, Japan

The increase of the coupling efficiency of a magnetostriction SH-wave electromagnetic acoustic transducer (EMAT) has been achieved by applying an angled bias magnetic field. A generalized model involving piezomagnetic theory is presented to explain the physical principle of the EMAT. It indicates a dominant contribution of the piezomagnetic coefficient $e_{6 x}^{(M S)}$ and predicts an efficient generation of the SH waves with an angled bias magnetic field. Dependences of the SH-wave amplitude on the magnitude and direction of the bias field were measured, which showed good agreement with those of the coefficient $e_{6 x}^{(M S)}$. The most efficient generation was accomplished with a $45^{\circ}$ angled bias field. The amplitude gain was $250 \%$.

KEYWORDS: electromagnetic acoustic transducer, magnetostriction, piezomagnetic effect, surface shear wave, coupling efficiency, angled bias field 


\section{Introduction}

Electromagnetic acoustic transducers (EMATs) are capable of generating and detecting elastic waves in electrically conductive materials or piezomagnetic materials without any mechanical contacting on the specimens [1-5]. They use the energy transfer between electromagnetic and acoustic fields, making the noncontact measurements possible. Among the variety of EMATs, the SH-wave EMAT activated by the magnetostriction effect has received attention $[6,7]$, because it generates and detects surface shear-horizontal $(\mathrm{SH})$ waves of high frequencies, which are useful for inspecting surface cracks.

The EMAT consists of a meander-line coil and a bias magnetic field applied along the straight parts of the coil, as illustrated in Fig. 1. Driving the meander-line coil with tone bursts, the surface (or plate) SH waves are generated for both sides via the piezomagnetic effect. Since the wavelength equals that of the meander-line period, the SH-wave frequency can be increased by decreasing the meander-line period. Recent advancement in printing circuit technique [8] enables us to fabricate a meander-line coil with a very small period, less than $10 \mu \mathrm{m}$, corresponding to the $\mathrm{SH}$-wave frequencies higher than $320 \mathrm{MHz}$ for standard steel. Thus, the ability of generating high-frequency surface $\mathrm{SH}$ waves is the most important advantage of this EMAT. However, low efficiency of the transfer with the EMAT has failed to make it a frequent replacement for a conventional piezoelectric transducer in practical applications. 
As shown by Thompson [6] and Igarashi et al. [7], a large bias field $\left(\sim 10^{5} \mathrm{~A} / \mathrm{m}\right)$ is needed for the maximum efficiency with the EMAT for standard steels. This is sometimes unrealistic for a thick specimen. However, it is experimentally known that an angled bias magnetic field increases the efficiency [9]. Thus, by controlling the direction and strength of the bias magnetic field, we shall achieve a much higher efficiency. Such an intentional increase of the efficiency requires an understanding and modeling of the physical principle of the EMAT. Several studies have been performed for investigating the magnetostriction effect on EMAT efficiencies [1-4], but no study has presented a generalized theory with a systematic measurement to confirm it. This is our goal.

First, we present a model for the piezomagnetic coupling to cause the magnetostriction body forces in a ferromagnetic material in the presence of an angled bias magnetic field. Second, we measure the dependences of the amplitude of the generated $\mathrm{SH}$ plate waves $\left(\mathrm{SH}_{0}\right.$ mode) on the strength and direction of the bias magnetic field. The measurements essentially confirm the theory, although they suggest the necessity of considering the electromagnetic loss in the low field region to achieve quantitative agreement.

\section{Theory}

\subsection{Magnetostriction forces}

When an external magnetic field is applied to a ferromagnetic material, a dimensional 
change arises depending on the magnitude and direction of the field. The normalized dimensional change is called magnetostriction. Magnetostriction occurs because, in iron for instance, the external magnetic field affects the $3 d$ subshell and changes the shape and size of the $3 d$ - electrons orbits to keep the minimum energy in the presence of the field. There is an elastic strain to achieve a balance between an atom's binding force and magnetic force in equilibrium even without any external field, which is the spontaneous magnetostriction and is equivalent to an eigenstrain in individual magnetic domains. In iron, a positive spontaneous magnetization exists along the domain's magnetization directions oriented to easy axes $<100\rangle$ [10].

In polycrystalline materials, the magnetostriction response to the external field is more complicated. The dimensional change occurs in two steps. Again we consider polycrystalline iron. First, the domains expand the volume, whose magnetization is oriented near to the external field, causing the positive dimensional change (elongation) along the field because of the positive spontaneous magnetostriction. After this domain's rearrangement, the magnetization rotates about the easy axis within the domain, which reduces the dimension due to the rotation of the spins. Thus, the magnetostriction along the applied field, $\varepsilon \mathrm{M}$, is a function of the field $H$ and the $\varepsilon_{\mathrm{M}}-H$ curve, or the magnetostriction curve, shows a maximum as will be shown in Fig. 5(a). The magnitude of magnetostriction is of the order of $10^{-6}$ for steels and $10^{-5}$ for nickel. 
Coupling between the elastic and magnetic fields for such a ferromagnetic material can be assumed to take a form similar to that of a piezoelectric material [11]:

$$
S_{I}=d_{I j}^{(M S)} H_{j}+s_{I J}^{H} \sigma_{J} \quad(I, J=1,2, \ldots, 6 ; j=x, y, z)
$$

Here, $S_{I}$ is a component of the engineering strain, $s_{I J}^{H}$ is a component of the compliance matrix at a constant field $H$, and $\sigma_{\mathrm{J}}$ is the stress component in contracted notation. $d_{I j}^{(M S)}$ can be given as

$$
d_{I j}^{(M S)}=\left.\left(\frac{\partial S_{I}}{\partial H_{j}}\right)\right|_{\sigma},
$$

which are piezomagnetic strain coefficients. When we apply a magnetic field to a ferromagnetic material in the stress-free state, the strain $S_{I}=d_{I j}^{(M S)} H_{j}$ will appear. The same strain field occurs with the stress field $\sigma_{K}=c_{K I}^{H} S_{I}$ without the magnetic field, where $c_{K I}^{H}$ is the elastic-stiffness coefficient at a constant field. The equivalent stress to cause the magnetostriction is $\sigma_{K}=c_{K I}^{H} d_{I j}^{(M S)} H_{j}$. If the applied magnetic field changes very quickly or is oscillated with a high frequency, the strain will fail to respond simultaneously with the field and the stress field $-\sigma_{K}$ will occur inside the material, which is the magnetostriction stress.

We can thus define the magnetostriction stress as

$$
\sigma_{I}^{(M S)}=-c_{I J}^{H} d_{J j}^{(M S)} H_{j}=-e_{I j}^{(M S)} H_{j}
$$

with the converse piezomagnetic stress coefficients

$$
e_{I j}^{(M S)}=c_{I J}^{H} d_{J j}^{(M S)}=-\left.\left(\frac{\partial \sigma_{I}^{(M S)}}{\partial H_{j}}\right)\right|_{S}
$$


The constitutive equation among the stress, strain, and field is then given by

$$
\sigma_{I}=-e_{I j}^{(M S)} H_{j}+c_{I J}^{H} S_{J},
$$

Equation (5) is similar to that of the piezoelectric stress equation. The body forces caused by the magnetostriction stress are given by

$$
\left\{\begin{array}{l}
f_{x}^{(M S)}=\frac{\partial \sigma_{1}^{(M S)}}{\partial x}+\frac{\partial \sigma_{6}^{(M S)}}{\partial y}+\frac{\partial \sigma_{5}^{(M S)}}{\partial z} \\
f_{y}^{(M S)}=\frac{\partial \sigma_{6}^{(M S)}}{\partial x}+\frac{\partial \sigma_{2}^{(M S)}}{\partial y}+\frac{\partial \sigma_{4}^{(M S)}}{\partial z} \\
f_{z}^{(M S)}=\frac{\partial \sigma_{5}^{(M S)}}{\partial x}+\frac{\partial \sigma_{4}^{(M S)}}{\partial y}+\frac{\partial \sigma_{3}^{(M S)}}{\partial z}
\end{array} .\right.
$$

The acoustic fields generated by the magnetostriction forces can be calculated by involving eq. (6) in the equation of motion when the piezomagnetic coefficients $\mathbf{d}^{\text {(MS) }}$ and $\mathbf{e}^{(\mathrm{MS})}$ are known. They depend highly on the magnitude and direction of the applied field, but they can be estimated from the magnetostriction curve as follows.

\subsection{Estimation of piezomagnetic coefficients}

For simplifying the analysis, we use several approximations: (i) The electromagnetic and elastodynamic fields are variables in the two-dimensional space of the $x$ - $z$ plane (see Fig. 2); the half space of $z>0$ is filled with a ferromagnetic metal, in which the $x-y$ plane defines the interface with a vacuum. (ii) The magnetostriction causes no volume change (isovolume); this is true for a nontextured polycrystalline ferromagnetic material, because randomly oriented easy axes average out the anisotropic magnetostriction of individual magnetic 
domains [10]. (iii) The displacement current is neglected, because EMATs use relatively low frequencies (less than $20 \mathrm{MHz}$ ). (iv) All time-dependent quantities cause harmonic oscillation and involve the $e^{\mathrm{j} \omega t}$ factor, which we omit in equations. Quantities with subscript 0 are time-independent and homogeneous in the field. (v) An infinitely wide meander-line coil is located with a liftoff $h$. The straight parts are along the $y$ axis.

When an alternating current $I$ is applied to the meander-line coil, the electromagnetic fields occur and penetrate into the material. They interact with the bias magnetic field to cause the body forces, and then, to generate elastic waves. For the meaner-line coil shown in Fig. 2, the magnetic fields in the material take the form [1]

$$
\left\{\begin{array}{l}
H_{x}=\frac{2 I}{\pi a} \sin \left(\frac{\pi a}{D}\right) e^{-2 \pi h / D} e^{q \mathrm{z}} \cos \left(\frac{2 \pi}{D} x\right) \\
H_{z}=-\frac{\sqrt{2} \pi \delta}{D} \cdot \frac{2 I}{\pi a} \sin \left(\frac{\pi a}{D}\right) e^{-2 \pi h / D} e^{q \mathrm{z}} \sin \left(\frac{2 \pi}{D} x\right)
\end{array},\right.
$$

where $D$ denotes the meander-line period and $a$ the width of the meander-line wire. $q$ is determined by $q^{2}=(2 \pi / D)^{2}+2 \mathrm{j} / \delta^{2}$ with the electromagnetic skin depth $\delta$. Usually, $\left|H_{x}\right|>>\left|H_{z}\right|$ because $(2 \pi / D) \delta<<1$. The presence of the liftoff $h$ exponentially decreases the magnitude of the electromagnetic fields in the material.

When a homogenous bias magnetic field $H_{0}$ is applied along the $y$ axis, the longitudinal magnetostriction $\varepsilon_{\mathrm{M}}\left(H_{0}\right)$ appears along the field. (Note that neither the Lorentz force nor the 
magnetization force occurs in this case). The magnetostriction normal to the field will be $-\varepsilon_{\mathrm{M}}\left(H_{0}\right) / 2$ because of the isovolume dimensional change. When the dynamic field $H_{x}$ is added, the total field occurs along the direction inclined by $\theta$ about the $y$ axis and the principal strains arise along the directions parallel and normal to the total field. Thus, in the coordinate system where the $y^{\prime}$ axis is along the total field (Fig. 3(a)), the strain field will be of the form

$$
S_{2}^{\prime}=\varepsilon_{M}\left(H_{t}\right) \equiv \varepsilon_{t}, S_{1}^{\prime}=S_{3}^{\prime}=\varepsilon_{M}\left(H_{t}\right)=-\frac{1}{2} \varepsilon_{t} .
$$

Note that no shearing deformation arises in this coordinate system. The strain field in the original coordinate system is given by

$$
\left\{\begin{array}{l}
S_{1}=S_{1}^{\prime} \cos ^{2} \theta+S_{2}^{\prime} \sin ^{2} \theta \\
S_{2}=S_{1}^{\prime} \sin ^{2} \theta+S_{2}^{\prime} \cos ^{2} \theta \\
S_{3}=S_{3}^{\prime} \\
S_{6}=\left(S_{2}^{\prime}-S_{1}^{\prime}\right) \sin 2 \theta=\frac{3}{2} \varepsilon_{t} \sin 2 \theta
\end{array},\right.
$$

where $\theta=\tan ^{-1}\left(H_{x} / H_{0}\right)$. It is clear from eq. (2) that $d_{4 x}^{(M S)}=d_{5 x}^{(M S)}=0 . \quad$ Then, we have

$$
\left\{\begin{aligned}
d_{1 x}^{(M S)} & =\left.\left(\frac{\partial S_{1}}{\partial H_{x}}\right)\right|_{\sigma}=\frac{\partial}{\partial H_{x}}\left(S_{1}^{\prime} \cos ^{2} \theta+S_{2}^{\prime} \sin ^{2} \theta\right) \\
& =\frac{3 \varepsilon_{t}}{H_{0}} \cos ^{3} \theta \sin \theta+\gamma \sin \theta\left(-\frac{1}{2} \cos ^{2} \theta+\sin ^{2} \theta\right) \\
d_{2 x}^{(M S)} & =-\frac{3 \varepsilon_{t}}{H_{0}} \cos ^{3} \theta \sin \theta+\gamma \sin \theta\left(-\frac{1}{2} \sin ^{2} \theta+\cos ^{2} \theta\right) \\
d_{3 x}^{(M S)} & =-\frac{1}{2} \gamma \sin \theta \\
d_{6 x}^{(M S)} & =\frac{3 \gamma}{2} \sin 2 \theta \sin \theta+\frac{3 \varepsilon_{t}}{H_{0}} \cos ^{2} \theta \cos 2 \theta
\end{aligned}\right.
$$

where $\gamma=\left(\partial S_{2}^{\prime} / \partial H_{t}\right)_{\sigma}$ stands for the slope of the magnetostriction curve and it is measurable. 
Analogous calculation results in the coefficients relating to the $y$ and $z$ direction dynamic

fields:

$$
\left\{\begin{array}{l}
d_{1 y}^{(M S)}=d_{3 y}^{(M S)}=-\frac{1}{2} \gamma, d_{2 y}^{(M S)}=\gamma, d_{4 y}^{(M S)}=d_{5 y}^{(M S)}=d_{6 y}^{(M S)}=0, \\
d_{1 z}^{(M S)}=d_{3 x}^{(M S)}, d_{2 z}^{(M S)}=d_{2 x}^{(M S)}, d_{3 z}^{(M S)}=d_{1 x}^{(M S)}, d_{4 z}^{(M S)}=d_{6 x}^{(M S)}, d_{5 z}^{(M S)}=d_{6 z}^{(M S)}=0 .
\end{array}\right.
$$

Thus, the matrix $\mathbf{d}^{(\mathrm{MS})}$ takes the following form for the tangential bias field along the $y$ direction:

$$
\left[d_{I j}^{(M S)}\right]=\left[\begin{array}{ccc}
d_{1 \mathrm{x}} & d_{1 \mathrm{y}} & d_{3 \mathrm{x}} \\
d_{2 \mathrm{x}} & d_{2 \mathrm{y}} & d_{2 \mathrm{x}} \\
d_{3 \mathrm{x}} & d_{1 \mathrm{y}} & d_{1 \mathrm{x}} \\
0 & 0 & d_{6 \mathrm{x}} \\
0 & 0 & 0 \\
d_{6 \mathrm{x}} & 0 & 0
\end{array}\right] .
$$

EMATs often use a static magnetic field much larger than the dynamic fields, that is, $\theta \sim 0$.

Such a high-field approximation reduces eq. (12) to

$$
\left[\begin{array}{ccc}
0 & -\frac{\gamma}{2} & 0 \\
0 & \gamma & 0 \\
0 & -\frac{\gamma}{2} & 0 \\
0 & 0 & \frac{3 \varepsilon_{t}}{H_{0}} \\
0 & 0 & 0 \\
\frac{3 \varepsilon_{t}}{H_{0}} & 0 & 0
\end{array}\right] .
$$


Equation (13) is comparable with the piezoelectric strain coefficients of crystals, which belong to a hexagonal-6mm point group [11].

When the bias magnetic field is applied in an angled direction by $\phi$ about the $y$ axis as shown in Fig. 3(b), similar expressions for the piezomagnetic coefficients to eqs. (10) and (11) are obtained by replacing $\theta$ with $\theta+\phi, H_{0}$ with $H_{0} \cos \phi$, and $H_{x}$ with $H_{x}+H_{0} \sin \phi$. For example,

$$
d_{6 x}^{(M S)}=\frac{3 \gamma}{2} \sin 2(\theta+\phi) \sin (\theta+\phi)+\frac{3 \varepsilon_{t}}{H_{0} \cos \phi} \cos ^{2}(\theta+\phi) \cos 2(\theta+\phi) .
$$

The $y$-direction body force needed to generate the SH waves is

$$
f_{y}^{(M S)}=\frac{\partial \sigma_{6}^{(M S)}}{\partial x}+\frac{\partial \sigma_{4}^{(M S)}}{\partial z}=-e_{6 x}^{(M S)} \frac{\partial H_{x}}{\partial x}-e_{4 z}^{(M S)} \frac{\partial H_{z}}{\partial z},
$$

Here, we neglected the terms proportional to $\partial H_{z} / \partial x$ because $\partial H_{z} / \partial x<<\partial H_{x} / \partial z \approx \partial H_{z} / \partial z$. The first term on the right hand side of eq. (15) contributes to generating the surface or plate SH waves propagating along the $x$ direction with the $y$ polarization. The second term contributes to the bulk shear waves propagating in the thickness direction with the $y$ polarization, which is out of range of this study. Thus, the coefficient $e_{6 x}^{(M S)}=c_{66} d_{6 x}^{(M S)}$ is the key for the SH-wave generation. By "SH wave", we mean surface SH waves and SH plate mode waves. Both waves are generated by the shear stress $\sigma_{6}\left(=\sigma_{\mathrm{xy}}\right)$ and their efficiency is determined by $e_{6 x}^{(M S)}$.

Application of an angled bias magnetic field causes the Lorentz force normal to the specimen surface, which however fails to contribute to the shear-wave generation. 


\section{Measurement}

The specimen we used was an interstitial-free steel, measuring $100 \mathrm{~mm}$ by $100 \mathrm{~mm}$ by 1 mm. It was located in a water-cooled solenoid coil, which provided a homogeneous magnetic field parallel to the specimen surface up to $8 \times 10^{4} \mathrm{~A} / \mathrm{m}$. We used a printed meander-line coil with period $D=0.9 \mathrm{~mm}$ and line width $a=0.02 \mathrm{~mm}$. The specimen was rotatable so as to change the bias field direction. As shown in Fig. 4, the $\mathrm{SH}_{0}$-mode wave was generated by the EMAT and detected by a wedge-mounted piezoelectric transducer. (Dispersion relation for the $\mathrm{SH}$ plate mode predicts that only the $\mathrm{SH}_{0}$ mode is excited by this meander-line period and we recognized the observed signal as $\mathrm{SH}_{0}$ mode from its group velocity.) This period corresponds to the $\mathrm{SH}_{0}$ mode with frequency of $3.55 \mathrm{MHz}$. The coil was driven by tone bursts and the detected signal was fed to a superheterodyne spectrometer, which extracted the amplitude of the same frequency component as the driving bursts signal. The Hall sensor detected the static field parallel to the surface and semiconductor strain gauges measured the magnetostriction along the field. All measurements were carried out after demagnetizing the specimen.

\section{Results and Discussion}

With the dimensions of the meander-line coil $(D=0.9 \mathrm{~mm}$ and $a=0.02 \mathrm{~mm})$ and 
reasonable guessed values, $I=5 \mathrm{~A}$ and $h=0.2 \mathrm{~mm}$, we obtained $H_{x}=2.75 \times 10^{3} \mathrm{~A} / \mathrm{m}$ from eq. (7).

Figure 5(a) shows the measured magnetostriction curve. We found that it can be well approximated by the following function of the form as seen by the solid line in Fig. 5(a)

$$
\varepsilon_{M}=34 H^{0.2} \cdot 1.48^{-1.5(H+1)}-5.5,
$$

where $\varepsilon_{\mathrm{M}}$ is in unit of microstrain and $H$ in $10^{4} \mathrm{~A} / \mathrm{m}$. This formula was used to calculate the piezomagnetic coefficients.

Figure 5(b) compares the measurements with the calculated coefficient $e_{6 x}^{(M S)}$ when the bias field is applied parallel to the straight parts of the meander-line coil $(\phi=0)$. They are principally consistent, showing the peak near the maximum magnetostriction, the minimum at the zero magnetostriction at the field of $\sim 2.4 \times 10^{4} \mathrm{~A} / \mathrm{m}$, and the gradual increase at high fields. Thus, the theory essentially explains the measurements. There are, however, discrepancies in magnitude between the theory and measurements; the results of measurements are lower than those of calculations in low fields and larger in high fields. This is attributed to the electromagnetic losses, including the hysteresis loss due to irreversible movement of magnetic domains and the eddy current loss (or the Joule-heating loss). They occur when the ferromagnetic material is dynamically magnetized and are closely related to the susceptibility of the material [10]: High susceptibility causes high electromagnetic losses. Susceptibility is much higher and the losses are much more significant in low fields than in high fields.

Figure 6 shows the measurements of the field dependence of the SH-wave amplitude 
with various bias field directions $\phi$. By applying an angled bias field, the SH-wave amplitude increases to a large extent even at low fields. The most efficient generation was achieved by the bias field with magnitude near $3 \times 10^{4} \mathrm{~A} / \mathrm{m}$; the maximum amplitude is larger than that when $\phi=0^{\circ}$ (classic case) by a factor 2.5. Figure 7(a) shows the coefficient $e_{6 x}^{(M S)}$ as a function of $\phi$, showing a maximum near $\phi=45^{\circ}$, a similar trend to the measurements in Fig. 7(b). Thus, an angled static magnetic field is quite effective to increase the EMAT efficiency.

\section{Conclusion}

We have presented a generalized modeling of a magnetostriction EMAT. Concerning the SH-wave EMAT, the piezomagnetic coefficient $e_{6 x}^{(M S)}$ governs the wave generation. The dependences of the generated SH-wave amplitude on the strength and angle of the bias field were explained by this coefficient. The most efficient generation occurs with the bias field inclined by $45^{\circ}$; the amplitude was increased by a factor of 2.5 compared with the classical EMAT. The theory presented here is applicable to any other magnetostriction EAMT, although the piezomagnetic coefficients must be calculated in individual cases. 


\section{References}

[1] R. B. Thompson: Physical Acoustics, ed. R. N. Thurston and A. D. Pierce, (Academic Press, NewYork, 1990), Vol.19, p.157.

[2] R. B. Thompson: IEEE Trans. Sonics and Ultrason. SU-25 (1978) 7.

[3] A.Wilbrand: Review of Progress in QNDE, eds. D.O.Thompson and D.E.Chimenti (Plenum, New York, 1987), Vol.7, p. 671.

[4] H. Ogi, M. Hirao and K. Minoura: J. Appl. Phys. 81 (1997), 3677.

[5] G. Alers and H. Ogi: EMAT Techniques, in Handbook of Elastic Properties of Solids, Liquids, and Gases, , eds. M. Levy, H. Bass, R. Stern and V. Keppens, (Academic Press, New York, 2001), Vol. 1, p. 263.

[6] R. B. Thompson: Appl. Phys. Lett. 34 (1979), 175.

[7] B. Igarashi, G. Alers and P. Purtscher: Proc. IEEE Ultrasonic Symp. (IEEE, New York, 1997) p. 709.

[8] H. Ogi, M. Hirao and T. Ohtani: IEEE Trans. Ultrason. Ferroelectr. Freq. Control 46 (1999) 341.

[9] G. Alers: personal communication.

[10] S.Chikazumi: Physics of Magnetizm (Wiley, New York, 1964).

[11] J. Nye: Physical Properties of Crystals (Oxford, Glasgow, 1957) p. 110. 
Fig. 1 Appearance of the SH-wave EMAT, which is activated by the magnetostriction effect.

Fig. 2 Two dimensional model of a meander-line coil.

Fig. 3 Inclined total magnetic field by (a) a bias field along the $y$ axis and (b) an angled bias field. The $x$ axis is selected so as to lie along the straight part of the meander-line coil.

Fig. 4 Generation of SH plate wave by a meander-line coil and its detection by a PZT transducer. $\phi$ denotes the angle between the bias magnetic field and straight part of the meander-line coil.

Fig. 5 (a) Magnetostriction curve of an interstitial-free steel. Open marks denote measurement values and solid line is the fitted function. (b) Field dependence of the $\mathrm{SH}$-wave amplitude and calculated piezomagnetic coefficient $e_{6 x}^{(M S)}$ for $\phi=0$.

Fig. 6 Field dependence of the SH-wave amplitude with angled bias magnetic fields.

Fig. 7 Dependences of (a) the piezomagnetic coefficient $e_{6 x}^{(M S)}$ and (b) the SH-wave amplitude on the direction of the bias magnetic field. 


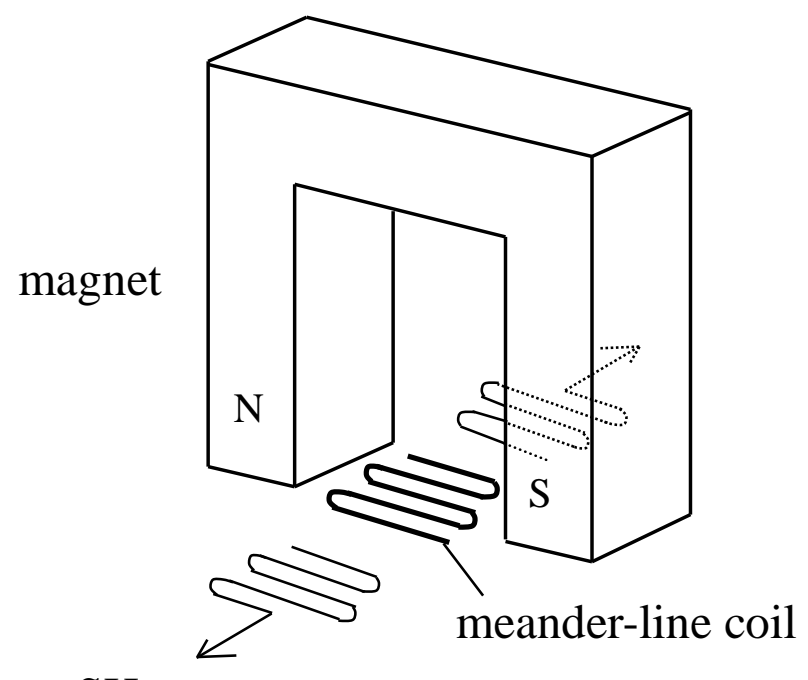

$\mathrm{SH}$ waves

Fig.1 


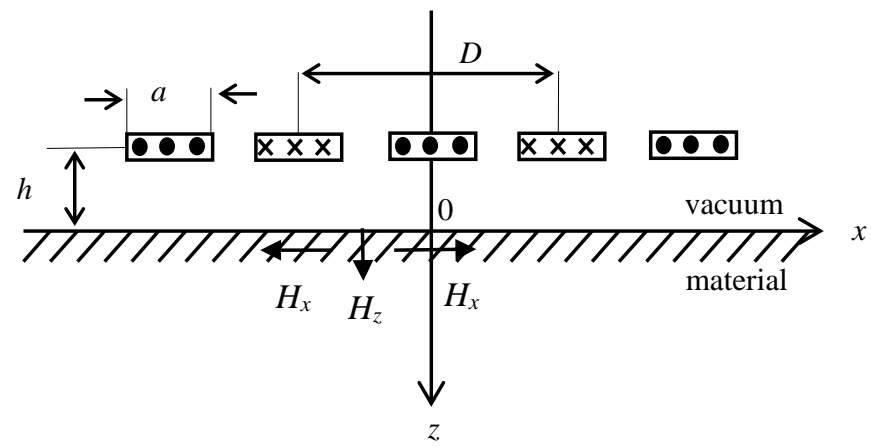

Fig. 2 


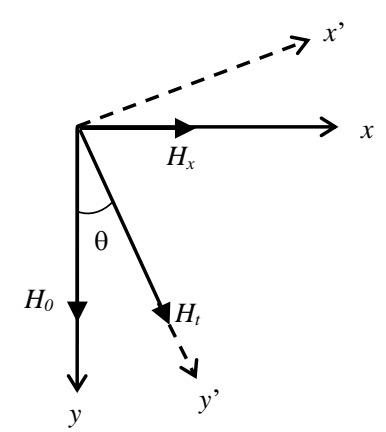

(a)

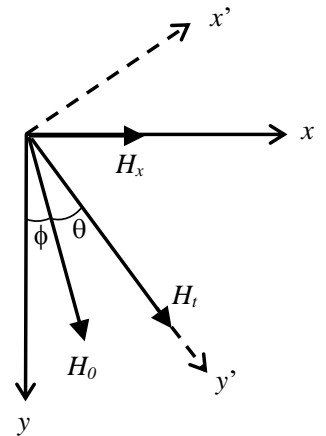

(b)

Fig. 3 


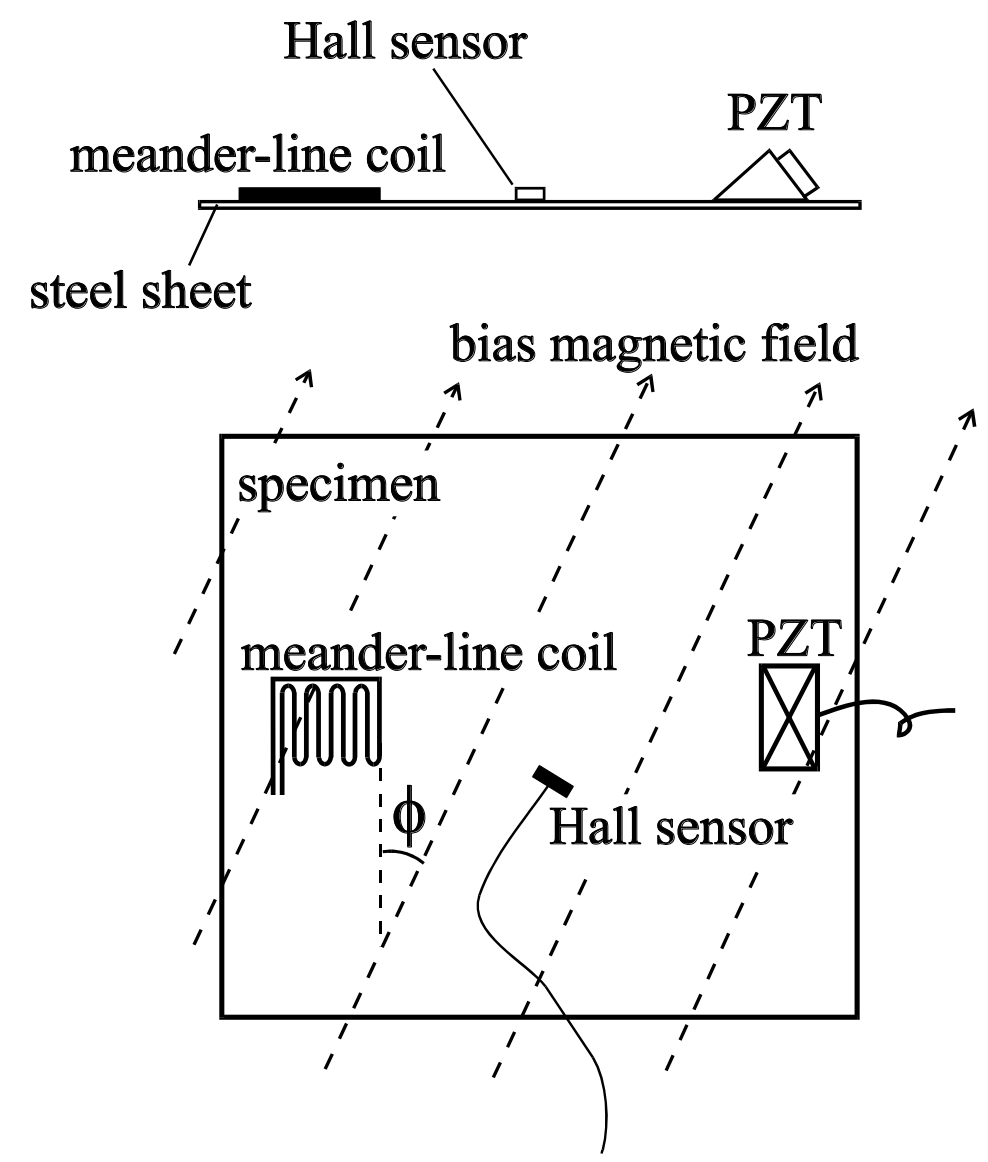

Fig. 4 


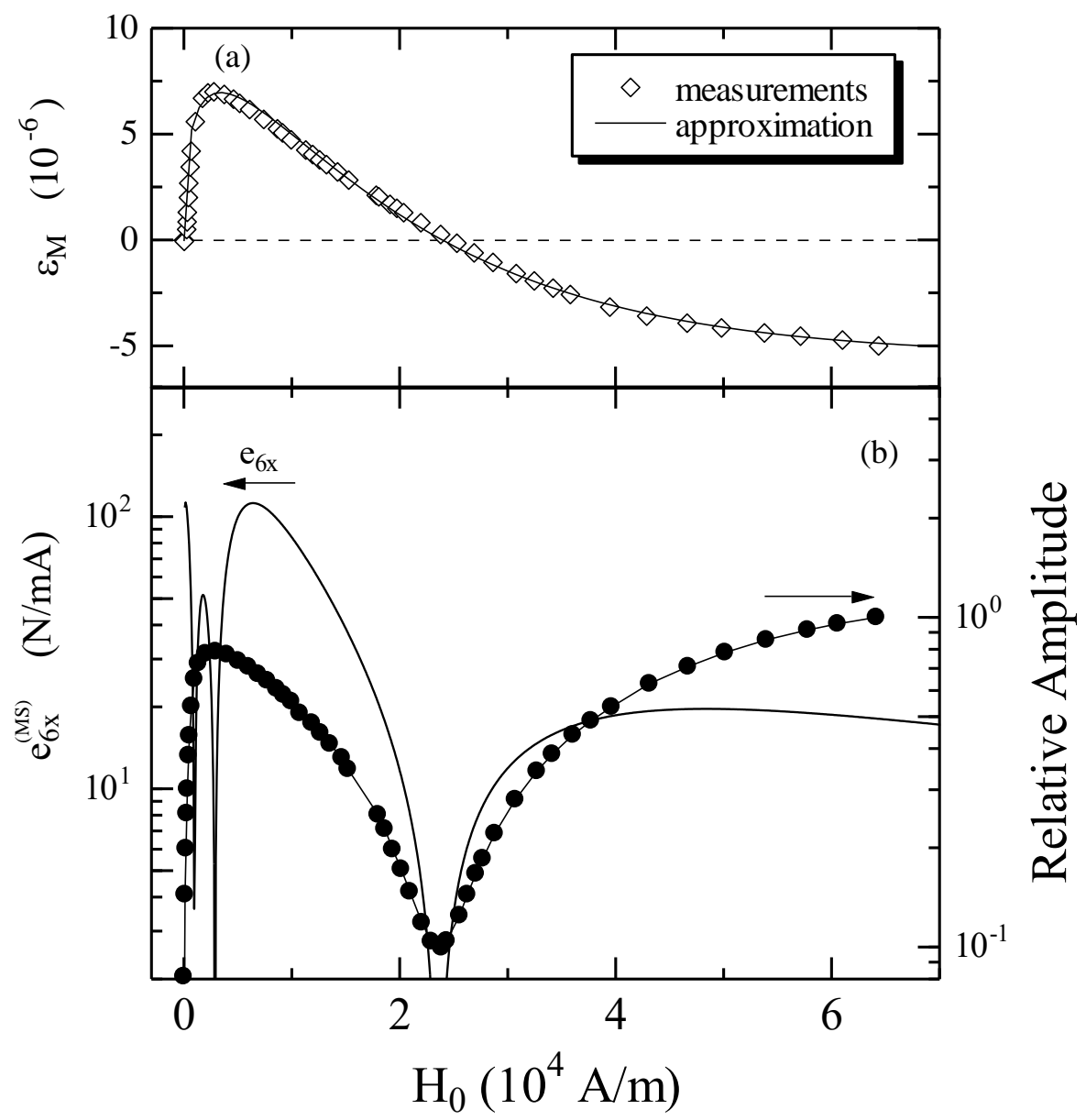

Fig. 5 


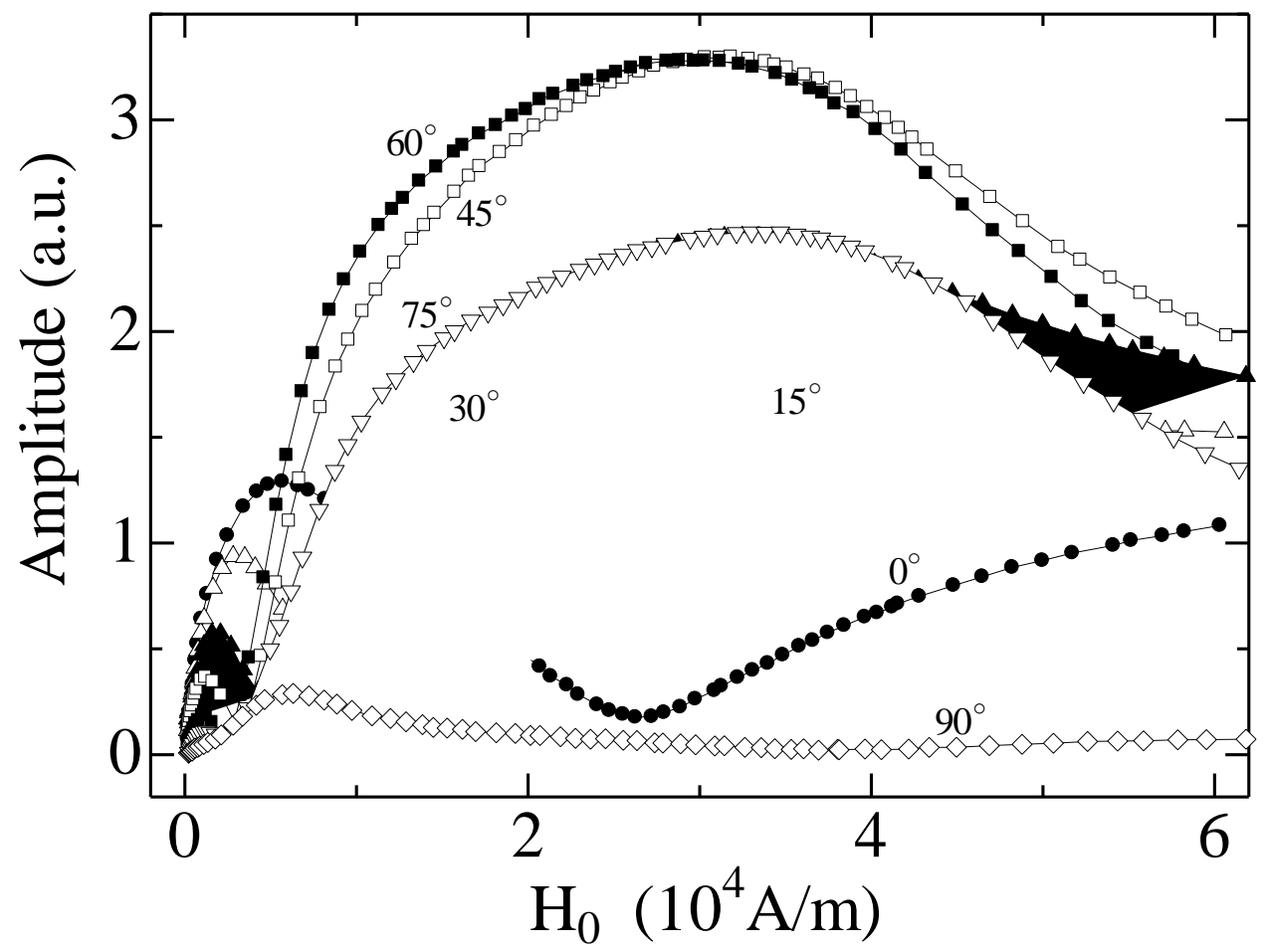

Fig. 6 


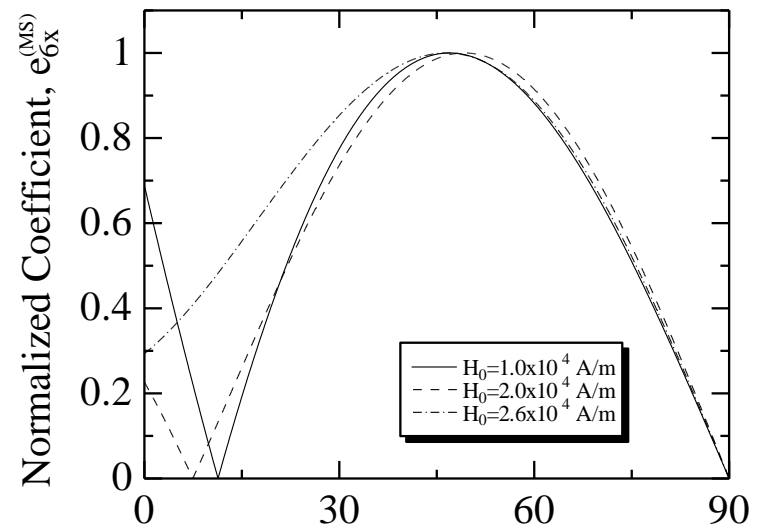

(a)

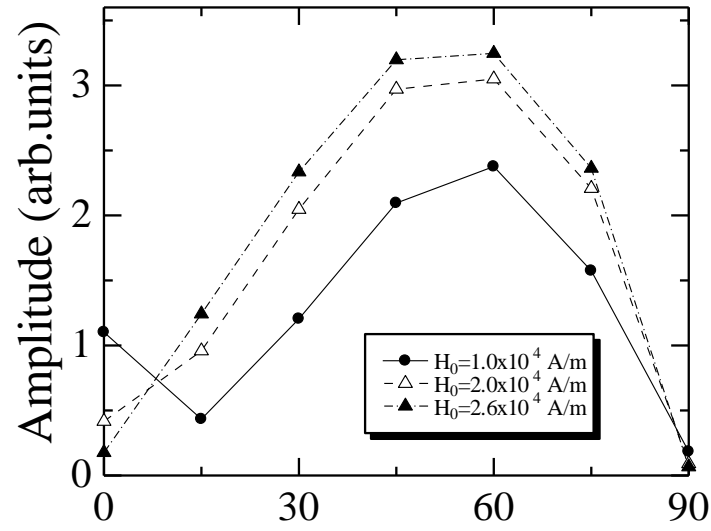

(b)

Fig. 7 